\title{
Exploration the Method of Low Dose Coronary Artery Imaging with Dual-Source CT
}

\author{
Zhiwei Huang, Bo Xiao, Lisha Zhong
}

Department of Biomedical Engineering, Luzhou Medical College, Luzhou, P.R.China

Email: hzwnet@126.com

Received 2013

\begin{abstract}
Objective: On the premise that the image quality meets the requirements of clinical diagnosis, we explored the methods to reduce the radiation dose of coronary artery imaging with Dual-Source CT (DSCT). Methods: We randomly selected 200 patients with coronary heat disease $(B I M<25 \mathrm{~kg} / \mathrm{m} 2)$, applied the scanning technology of regulating the dose of heart electric pulse (AUTO) on the 100 patients in group A. In this group for different heart rate we chose different full dose exposure time window. For the 100 patients in group B, we used conventional full dose (OFF) scan mode. The DSCT automatically selected the best time and phase to reconstruct the image. We used the 5 point system to evaluate the image quality, measured and compared the image noise and radiation dose. When $P<0.05$, the differences between the two groups have statistical significance. Results: The image quality scores between the two groups showed no significant difference $(P>0.05)$. The average image noise in group $A$ is $(41.76 \pm 7.98) \mathrm{HU}$, in group $B$ the average image noise is $(43.97 \pm 3.88) \mathrm{HU}$, the difference between the two groups was not statistically significant $(P>0.05)$. The average CTDIvol of group $A$ and $B$ were $(20.63 \pm 2.24) \mathrm{mGy}$, (38.11 \pm 10.69$) \mathrm{mGy}$, respectively, then $P<0.01$. The average DLP of group $A$ and $B$ are $(235.75 \pm 28.64) \mathrm{mGycm}$ and $(492.59 \pm$ 125.49) mGycm respectively, then $P<0.01$, the difference of radiation dose had statistical significance $(\mathbf{P}<\mathbf{0 . 0 5})$. Conclusions: For coronary artery imaging with DSCT the heart electric pulse (AUTO) regulation technology can meet the diagnostic requirements and effectively reduce the radiation dose.
\end{abstract}

Keywords: Dual-Source CT(DSCT); Coronary CT Angiography; Low dose; Noise;

\section{INTRODUCTION}

The study shows that the DSCT coronary artery imaging has a high degree of consistency in the diagnostic results of coronary stenosis and selective coronary arteriongraphy (SCA) ${ }^{[1]}$. DSCT evaluation on moderate to severe coronary artery stenosis has higher compliance and consistency with the SCA, DSCT can be used as a noninvasive conventional means in clinical screening and diagnosis ${ }^{[2]}$. DSCT has two sets of X-ray tubes and relative detectors, two sets of DAS systems are installed in 90 degree angle on the rack. Compared with multi-slice spiral CT (MSCT), DSCT reaches higher time resolution, its detector covers more areas, its scanning speed becomes faster, so the scan time is greatly shorten, and the radiation doses is significantly reduced by about $15 \%$ to $20 \%{ }^{[3]}$. It also has other advantages, such as the pitch can be automatically adjusted with the heart rate without the limit of the heart rate, and the negative prognosis is high ${ }^{[4]}$. With the appearance of DSCT, the radiation dose is reduced, but the radiation dose is still widely concerned. The heart disease committee declared that if 2000 patients receive CT examination with the radiation dose of $10 \mathrm{mSv}$, one of them will suffer from malignant tumors. For the overall risk of cancer caused by radiation, male is less than the women, and the gap between men of different ages, and there is little gap between men of different ages; but the overall cancer risk of women decreases with increasing age ${ }^{[5]}$. In this paper for coronary artery imaging with DSCT, we compared heart electric pulse (AUTO) with conventional full-dose (OFF) scan mode, compared the image quality, image noise, and radiation dose of the two sets. Ensuring the quality of the image to meet the diagnostic requirements, we tried to find methods to minimize the radiation dose.

\section{MATERIALS AND METHODS}

\subsection{Case Selection}

From May 10, 2012 to May 25, 2012, we randomly selected 200 patients from West China Hospital of Sichuan University, these patients were clinical suspected or diagnosed with coronary heart disease $(\mathrm{BIM}<25 \mathrm{~kg} / \mathrm{m} 2)$. 100 cases are in group A (63 cases are male, female 37 
cases are female, their ages were 34 - 82 years old, the average age was 58 years old ), and in their scanning mode, we used AUTO scanning mode. Another 100 cases are in group B (57 cases are male, 43 cases are female, their ages were 33 - 80 years old, their average age is 56.5 years old), we applied OFF scanning mode on them. Two groups of patients are not restricted by heart rate, age and gender, except serious arrhythmia or frequent premature beats patients. In both groups we used retrospective cardiac-gated imaging technique.

\subsection{Scanning Preparation}

We tried to understand whether patients are allergic to iodine, and their renal functions are normal. We also tried to eliminate the tension and depression of the patients. We conducted strict breath training on patients, and enjoined patients to keep respiratory amplitude consistent for every time. The patients are in the supine position, the feet of the patients entered the CT gantry first. ECG electrodes were correctly placed, and the leads with higher $\mathrm{R}$ wave were selected. For the patients without hypotension, we sprayed Nitroglycerin Aerosol 2-3 times under their tongues.

\subsection{Scanning Method}

\subsubsection{Scanning Operation}

We used the Siemens Somatom Definition as DSCT scanner. For the patients in group A and B, we applied the same time resolution, space resolution, rotation time $(0.33 \mathrm{~s})$, collimator width $(64 * 0.6 \mathrm{~mm})$, image reconstruction thickness $(0.75 \mathrm{~mm})$, reconstruction interval $(0.5 \mathrm{~mm})$. At the same time, the scanning range (from 1 to $2 \mathrm{~cm}$ under the bifurcation of trachea to diaphragmatic surface of heart), contrasted medium injection scheme, and image processing methods were kept consistent. We chose Iopamidol $(370 \mathrm{mgI} / \mathrm{ml})$ contrast agent and Germany binocular high-pressure syringe (Ulrich Medical), Syringe needle was placed on the forearm vein. Firstly physiological saline was injected, then contrast agent Iopamidol was injected, at last physiological saline was injected.

\subsubsection{Iodine Concentration Monitoring}

We used contrast agent tracer method. In the center of ascending aorta (it is under the tracheal bifurcation and away from the bifurcation $2-3 \mathrm{~cm}$ ), we chose the region of interest and detected the CT value, and the threshold value was set to $100 \mathrm{HU}$. When the CT values in the region of interest reach $100 \mathrm{HU}$, the CT will automatically trigger scan with a delay of 6s. Pay attention to avoiding the impact of the superior vena cava.

\subsubsection{ECG}

We scanned the patients after their heart rate was steady and used AUTO scanning mode: the exposure range of the full dose scan mode was a certain time window in the R-R interval, and the rest exposure dose was $1 / 4$ of the full dose. The time window of the full dose exposure was in the R-R interval, and this time window corresponded to different heart rate range. For relatively fast or slow heart rate, the full-dose exposure range in R-R interval was the smallest. For normal heart rate, full-dose exposure range was the maximum. OFF scanning mode: the full dose-exposure range is the entire R-R interval.

\subsubsection{Image Post-processing}

We adopted Siemens Syngo Miltimodality Workplace as the workstation software for automatically selecting the best time and phase. By calculating the movement speed of each vessel coronary, the best time and phase were selected for reconstruction of $0.75 \mathrm{~mm}$ thickness, so we got the best coronary artery images at systole and diastole. We used the software Circulation to perform the image post-processing, main technologies includes: Maximum Intensity Projection, Curved Surface Reconstruction), Volume Reproduction in order to multi-facedly show the left main of coronary artery and its main branches Right Coronary Artery, Anterior Descending and Circumflex Artery.

\subsection{Image Quality Assessment and Radiation dose Calculation}

\subsubsection{Image Quality Assessment}

Two experienced diagnostic physicians rated images by using the double-blind method. For the inconsistent judgments, they jointly and secondly read the film to reach agreement. We used 5-point scale in the grading of the image quality. The standard of 5 points is that the image can be display clearly without artifacts, the blood flow filling in all the vascular is good. The standard of 4 points is that there is minor artifacts, only a certain section of the main coronary artery is slightly fuzzy without split-level or ladder-like artifacts, the diagnosis is not affected. The standard of 3 points is that in the film the artifacts are medium, more than $1 / 2$ of one coronary artery trunk is fuzzy, but we can diagnose the illness. The axial image is rather vague, but the reconstructed image can be used for diagnosis. The standard of 2 points is that in the film the artifacts are severe, all the parts of one coronary artery are fuzzy or discontinuous, parts of the blood vessels have split-level artifacts, and the diagnosis is limited. The standard of 1 point is that the coronary artery can not be recognized, the tube pulse can not be analyzed, and we can not diagnose the illness. If the score of the image quality can reach 4-5 points, the image can be considered to be a high-quality image; if the score is 3 points, the image is evaluated image; if the 
score is 1-2 points, the image has severe artifacts, it can not be evaluated and is poor image ${ }^{[6]}$.

\subsubsection{Radiation dose and Image Noise}

The dose of X-ray is provided by the equipment. ED is the effective dose of radiation in the patient, and it can be calculated by using the formula $\mathrm{ED}=\mathrm{DLP} \times \mathrm{k}$, where $\mathrm{k}=0.017^{[7]}$ and DLP is the dose length product. DLP (myGycm) is displayed on the device. CTDIvol is CT dose index, it is also displayed on the device. We set $1 \mathrm{~cm}^{2}$ region of interest (ROI) which is put on aortic center, the ROI is on the beginning part of the left and right coronary artery and it is also on the level aortic root. We measured the ROI's CT value, used the values' standard deviation as the image noise. We calculated the average value of the twice CT values in the left and right coronary artery, and used this average value as the ultimate CT value. Similarly, the average value of the standard deviation was calculated twice.

\subsection{Statistical Method}

We analyzed the obtained data by using software SPSS11.5. We compared the scores' differences of image quality between two groups by applying the values of $x^{2}$. We also applied two independent samples t to test and compare the radiation dose and image noise. If $\mathrm{P}<0.05$, we thought the difference had statistical significance $^{[8]}$.

\section{RESULTS}

\subsection{Image Quality}

In Group A, we got 76 cases, for which the score of image quality was 5 point, accounting for $76 \%$. At the same time we got 4 points in 20 cases, accounting for $20 \%$ and 3 points in 4 cases, accounting for $4 \%, 2$ points and 1 point, 0 cases, accounting for $0 \%$. In group $\mathrm{B}$, we got 5 points in 84 cases, accounting for $84 \%$ and 4 points in 14 cases, accounting for $14 \%$, 3 points in 2 cases, accounting for $2 \%, 2$ points and 1 point 0 cases, accounting for 0\% (Shown in Table 1). For all the coronary artery images whose quality can be evaluated, the difference of scores between two groups showed no significant difference $(\mathrm{P}>0.05)$.

Table1. The comparison of the image quality in Group A and B.

\begin{tabular}{cccccccc}
\hline Group & 5 points & 4points & 3points & 2points & 1point & $x^{2}$ & $P$ \\
\hline Group A & 76 & 20 & 4 & 0 & 0 & & \\
& & & & & & 2.215 & 0.346 \\
Group B & 84 & 14 & 2 & 0 & 0 & & \\
\hline
\end{tabular}

\subsection{Image Noise}

The average CT value in Group A is $(475.1 \pm 45.75) \mathrm{HU}$, the average CT value in Group B is $(451.1 \pm 45.77) \mathrm{HU}$, $\mathrm{P}>0.05$. The average image noise in Group $\mathrm{A}$ is (41.76 \pm 7.98) $\mathrm{HU}$, the average image noise in Group $\mathrm{B}$ is (43.97 \pm 3.88) HU, $\mathrm{P}>0.05$. The difference of image nose between Group A and B had no statistical significance, as shown in following Table 2.

\subsection{Radiation Dose}

The radiation dose in the process of cardiac coronary artery examination consists of the following 3 parts: Scan, Contrast Agent Tracer Scan and Enhanced Scan. The radiation dose is the sum of these three parts. We did statistical analysis, and calculated CTDIvol, mean dose length product ( $\triangle \mathrm{DLP}$ ) and $\mathrm{ED}$, we also compared the results. In Group A, the average CTDIvol is (20.63 \pm 2.24) $\mathrm{mGy}$, in Group B the average CTDvol is (38.11 \pm 10.69) $\mathrm{mGy}$, it reduced $(17.48 \pm 8.45) \mathrm{mGy}$, and it decreased by nearly 1 time, $\mathrm{P}<0.05$. In Group $\mathrm{A}$, the $\triangle$ DLP is (235.75 \pm 28.64$) m G y c m$, in Group B the $\triangle \mathrm{DLP}$ is $(492.59 \pm 125.49) \mathrm{mGycm}$, it reduced $(256.84 \pm 96.85)$ mGycm, it decreased more than 1 time, $\mathrm{P}<0.05$. In Group A the average ED is $(4.01 \pm 0.49) \mathrm{mSv}$, in Group $\mathrm{B}$ the average ED is $(8.37 \pm 2.13) \mathrm{mSv}$, it reduced $(4.36$ $\pm 1.64) \mathrm{mSv}$, it decreased more than 1 time, $\mathrm{P}<0.05$. The radiation dose difference between two groups was statistically significant, as shown in Table 3.

\section{DISCUSSIONS}

DSCT coronary artery imaging in the diagnosis of cardiovascular diseases has unique advantages, more and more clinical cardiologist take CT as an important

Table 2. Comparison of the image noise in Group A and B.

\begin{tabular}{ccc}
\hline & CT Value $(\mathrm{HU})$ & Noise $(\mathrm{HU})$ \\
\hline Group A & $475.1 \pm 45.75$ & $41.76 \pm 7.98$ \\
Group B & $451.1 \pm 45.77$ & $43.97 \pm 3.88$ \\
Value of t & 0.829 & -0.557 \\
Value of $\mathrm{p}$ & 0.431 & 0.593 \\
\hline
\end{tabular}

Table 3. Comparison of the radiation dose of the Group A and B

\begin{tabular}{cccc}
\hline & CTDIvol(mGy) & $\triangle \mathrm{DLP}(\mathrm{mGycm})$ & $\mathrm{ED}(\mathrm{mSv})$ \\
\hline Group A & $20.63 \pm 2.24$ & $235.75 \pm 28.64$ & $4.01 \pm 0.49$ \\
Group B & $38.11 \pm 10.69$ & $492.59 \pm 125.49$ & $8.37 \pm 2.13$ \\
Value of $\mathrm{t}$ & -16.007 & -19.954 & -19.954 \\
Value of $\mathrm{p}$ & 0.000 & 0.000 & 0.000 \\
\hline
\end{tabular}


examination tool for non-invasive coronary artery imaging, it can be used as a supplement Cardiovascular Angiography, sometimes it can even replace Cardiovascular Angiography. In the process of the entire examination of DSCT, X-ray dose is large, the radiation hazards it produces gets more and more attention. In order to follow the principle of ALARA (as low as reasonable achievable), many scholars have done a lot of research in this respect, including hardware, software and optimizing programs of scan, such as Heart Bowtie, ECG Current Control, Cardiac noise subtraction filter, etc ${ }^{[9]}$. But the factors which affect the image quality are complex, some of these factors are closely related and influence each other. Some parameter settings may improve the image quality, but on the other hand, they may reduce the image quality in another aspect. So we must comprehensively consider, and separately set the parameters of scan according to the different clinical needs. But it is difficult for us to balance the various factors to obtain high-quality images in order to improve the diagnostic rate. Currently, there is no standard for quality control in coronary artery imaging of Dual-Source CT in our country.

So far, people have paid more and more attention to the potential hazards of ionizing radiation in CT. When ionizing radiation is working on human body, it can produce biological impacts and do harm to human body. The break of the structure of DNA double helix in human body is the critical damage to the cells. Radiation induces gene mutations or the break of the double helix structure, the distortion increases, and eventually, the radiation can lead to cancer ${ }^{[10]}$. The reports pointed out that for every more radiation dose of $10 \mathrm{mSv}$, the mortality rate will increase by $0.04 \%{ }^{[11]}$. The actual radiation dose of CT examination is 2-5 times the dose of effective radiation ${ }^{[12]}$. The dose of effective radiation mainly comes from the enhanced period. In the aspect of optimizing scanning program, people mostly focus on controlling the radiation dose of enhanced period. In most cases, the image quality and the radiation dose have the inverse relationship. To balance the relationship between them, we need to change the fixed scan mode. In practical application, we should pay attention to the individual conditions of the different patients, such as the size of the heart, breast size of the female patients and cardiothoracic ratio, etc. According to these conditions, we determine the appropriate scan mode and scan parameters. So we can achieve the personalized and reasonable scanning. A recent survey, which is an international research for radiation dose in coronary artery CTA, displays that the radiation dose in different hospitals have obvious gap, from an average of $5 \mathrm{mSv}$ to an average of $30 \mathrm{mSv}$. This demonstrates that reasonable scan mode can effectively reduce the radiation dose ${ }^{[13]}$. Therefore, on the premise that the image quality meets the requirements of clinical diagnosis, it becomes important for us to effectively reduce the radiation dose in the CTA examination of coronary artery, and this can reduce the radiation damage caused by human factors.

Reducing the radiation dose is generally divided into two categories: new low-dose technology and optimizing scan parameters. The new scanning technology uses the ECG current modulation technique, Prospective ECG Trigger Scan Technology and noise reduction algorithms. The methods of optimizing scan parameters are lowering tube current, lowering tube voltage, reducing the scan rage of Z-axis and reducing the number of scans based on body mass or body mass index (BMI). In this article, in order to reduce the radiation dose, we used the technology of regulating the dose of heart electric pulse (AUTO), and this technology belongs to retrospective cardiac-gated imaging technique. Through the study and analysis, we concluded that the regulation technology of dose of heart electric pulse (AUTO) can make the image quality completely meets the requirements of clinical diagnosis, and compared with the conventional full dose (OFF), this technology can significantly reduce the radiation dose of $(4.36 \pm 1.64) \mathrm{mSv}$, the radiation dose decreased more than 1 time. Of course, it also has inadequacies: 1. Data acquisition is not carried out throughout the whole cardiac cycle, and we can not analyze the cardiac function. 2. If the patient's heart rate in unstable, it is easy for us to select the wrong full-dose regions. 3. In the non-full-dose exposure regions of the R-R interval, the image quality is a little bit worse than that of the image in full-dose regions. 4. If there are heart premature beats in the scan, we can not edit the ECG.

The study has the following limitations. The individualized scan mode according the patient's BMI was not adopted, the radiation dose still has some space to be reduced, and in this aspect the study should be continued. If the post-processing of image only uses one of the two periods in image reconstruction, we can only reconstruct the image of one period, therefore the radiation dose will be reduced by 1 time, and in this aspect can be studied much further.

\section{REFERENCES}

[1] Lei ZHUANG, Jihong GAN, Jianjun LIU. Diagnosis of coronary artery disease by dual source CT coronary angiography. Medical Journal of National Defending Forces in Northwest China, 2010, 31 (4): 273-275.

[2] Yuan Zhao, Hong Pu. Comparatively Studying the Application of Dual Source CT in Coronary 
Angiography. Journal of Sichuan Continuing Education College of Medical Sciences. 2009, 28 (2): 87-89.

[3] Yan Gao. Exploration of the low dose technique in the examination of the coronary arteries angiography with dual-source CT. Sichuan Medical Journal, 2009, 30(12):1967-1969.

[4] Chiwei Wang. The current study of Dual-source CT coronary artery imaging. Anhui Medical Journal, 2010, 31(10): 1256-1258

[5] Renwei Liu. Research Progress of Dual-source Computed Tomography (DSCT) Low-dose Coronary Angiography. Medical Recapitulate, 2009, 15(21):3317-3320.

[6] Jiong Li. The application of optimizing scan parameters of Dual-Source CT in coronary artery imaging for reducing radiation dose. Nei Mongol Journal of Traditional Chinese Medicine, 2012, 10:82-83.

[7] Lifeng Zhang, Maoyi Zhou, Li Yang. Influence of coronal arterial image quality of 64 detector row CT in patients with ECG-gated scanned and optimal R-R phase imaging reconstructed. Chinese Journal of Medical Imaging Technology, 2008, 24(1):88-91.

[8] Wanshi Zhang, Jiaxing $\mathrm{Xu}$. See Development of Low-dose CT from RSNA 2008. China Medical Device Information, 2009, 7(1):12-13
[9] Aijun Wang, Jianguo Zhao, Xuejun Ping. The comparison of two different methods of coronary artery imaging in Dual-Source CT. Journal of Ningxia Medical College, 2010, 32(3): 443-445.

[10] Zixu Yan, Zhaoqi Zhang. Low tube voltage in reduction of radiation dose in dual-source CT coronary angiography. Chinese Journal of Medical Imaging Technology, 2009, 25(9): 1614-1616.

[11] Weiwei Qi, Xiangke Du, Ying Guo. A method to optimize the selection of tube current for consistent image noise and dose control in 64-slice spiral helical CT cardiac imaging. Image Technology, 2008, 452(10):1026-1030.

[12] Xiaoyang Chen, Jiankun Zhai, Hui Li. Clinical application and radiation prevention of CT scanning technology. China Modern Medicine, 2010, 17(8):61-62.

[13] Gou Wenjing, Shiyuan Liu, Hong Yu. Strategy and research progress in coronary computed tomography angiography with low-dose scan. International Journal of Medical Radiology, 2012, 35(2): 147-150.

[14] Hausleiter J, Meyer T, Hermann F. Estimated radiation dose associated with cardiac CT angiography. JAMA， 2009, 301:500-700.

[15] Yan Qi, Shuang Qi. The application study of Dual-Source CT in cardiac imaging. Chinese Heart Journal, 2010, 22(2):302-304. 